\title{
長さの異なる二個の平行き裂を有する圧電厚板の電気熱弾性応答*
}

$$
\begin{array}{llllll}
\text { 上田 } & \text { 整*1, 石 井彰 } & \text { 人 }^{* 2} \\
\text { 近 } & \text { 藤 } & \text { 宏 } & \text { 憲*3, 井 川 } & \text { 健*3 }
\end{array}
$$

\section{Thermoelectromechanical Response of a Piezoelectric Strip with Two Parallel Cracks of Different Lengths}

\author{
Sei UEDA*4, Akito ISHII, \\ Hironori KONDO and Ken IKAWA \\ ${ }^{* 4}$ Department of Mechanical Engineering, Osaka Institute of Technology, \\ 5-16-1 Omiya, Asahi-ku, Osaka-shi, Osaka, 535-8585 Japan
}

\begin{abstract}
The thermoelectromechanical fracture of a piezoelectric material strip with two parallel cracks of different lengths under electric and thermal loadings is considered. The crack faces are supposed to be insulated thermally and electrically. Fourier transform techniques are used to reduce the mixed boundary value problems to two systems of singular integral equations. The singular integral equations are solved by using the Gauss-Jacobi integration formula. Numerical calculations are carried out, and detailed results are presented to illustrate the influence of the parameters of indicating the crack lengths, the crack location and the crack spacing on the stress and electric displacement intensity factors. The results for the temperature and thermal stress distributions are also included. From the numerical results, we can find that the normalized intensity factors are under the great influence of the geometric parameters. Especially, the interaction between two different length cracks is complicated.
\end{abstract}

Key Words: Stress Intensity Factor, Elasticity, Thermal Stress, Fracture Mechanics, Piezoelectric Material, Two Parallel Cracks, Integral Transform

\section{1. 緒言}

圧電材料は，忘答速度および変形制御に優れること から、坚やサアクチュエータとして用いられている。 この特性を活か寸ことにより，温度変化に起因寸る熱 変形を適切に制御できる知的材料システムの構築が可 能となる(1).このため, 圧電材料を用いた知的材料シ ステムの最適設計・開発を目的とした電気熱弾性挙動 の解明が要望され，多くの解析結果が報告されている (2) (4).さらに, 知的材料システムの健全性評価に仗, 熱的環境下にある⿸厂電材料を対象とした笔気熱弾性破 壊力学的挙動の解明も重要であり, Wang and Mai ${ }^{(5)}$ および著者ら(6) (8) は, 圧電材料を対象に電気的熱 的負荷を受ける場合の特異電気熱弾性場を理論解析し ている。

一方，材料内には多数のき裂が存在し，それらの 相互干渉により破壊にいたると考えられる．従って， き裂間相互干涉の影響を解明することも重要であり， 二個の等長き裂を対象とした研究が報告されている

\footnotetext{
* 原稿受付 2008 年 2 月 25 日.

*1 正員, 大阪工業大学工学部 (祭 535-8585 大阪市旭区大宮 516-1).

*2 准員, 大阪工業大学大学院.

*3 正員, 大阪工業大学大学院.

E-mail : veda@med.oit.ac.jp
}

(9) (11). しかし，奏際上重要な長さの異なるき裂を対 象とした研究例は見当たらないようである.

そこで本報では，長さの異なる二個の平行き裂を有 寸る圧電厚板を対象に, 電気的負荷および熱的負荷が 作用寸る場合を考え，き裂先端の特異場を理論解析し た. 解析には, Fourier 変換法および熱弾性変位ポテン シャル法を用い, 問題の解を特異積分方程式の解に帰 着して解く方法を採用した (12),(13)。 また，特異積分 方程式の数値解析には, Gauss-Jacobi の数値積分公式 （14）を用い，忘力・電束密度拡大係数に及ぼす幾何学 的形状および電気的熱的負荷の影響を明らかにした。

\section{2. 問題の設定と基碮式}

図 1 に示す直角座標系 $(x, y, z)$ において, 厚さ $h=$ $h_{1}+h_{2}$ の圧電厚板を考え, $z= \pm h_{0}$ 面上に長さ $2 c$ および $2 \mu c(\mu \geq 0)$ のき裂が自由表面に平行に存在す るものとし，平面ひずみを仮定する．圧電厚板の上面 $\left(z=h_{1}\right.$ 面) および下面 $\left(z=-h_{2}\right.$ 面) の温度がそれ ぞれ $T_{10}, T_{20}$ に保持され， $z$ 軸の正の向きに一様電束 密度 $D_{0}$ が作用する問題を設定し，分極方向を $z$ 軸方 向とする. なお，弾性定数を $c_{j k}$, 圧電定数を $e_{j k}$, 誘 電定数を $\varepsilon_{j j}$, 熱弾性定数を $\lambda_{j j}$, 熱伝導率を $\kappa_{x}, \kappa_{z}$ 
㧍よび焦電定数を $p_{z}$ とし, 下添字 $x . y . z$ は座標軸方 向を示すものとする.

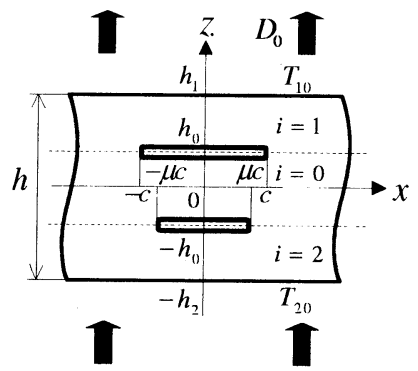

Fig.1 Two parallel cracks in a piezoelectric strip.

温度を $T_{i}(x, z)$, 変位ベクトルの成分を $u_{x i}(x, z)$, $u_{z i}(x, z)$, 静電ポテンシャルを $\phi_{i}(x, z)$ と寸ると, 応力 テンソルの成分 $\sigma_{x x i}(x, z), \sigma_{z z i}(x, z), \sigma_{z x i}(x, z)$ およ び電束密度ベクトルの成分 $D_{x i}(x, z), D_{z i}(x, z)(i=$ $0.1,2)$ は次のように与えられる(17).

$$
\left.\begin{array}{rl}
\sigma_{x x i} & =c_{11} \frac{\partial u_{x i}}{\partial x}+c_{13} \frac{\partial u_{z i}}{\partial z}+e_{31} \frac{\partial \phi_{i}}{\partial z}-\lambda_{11} T_{i} \\
\sigma_{z z i} & =c_{13} \frac{\partial u_{x i}}{\partial x}+c_{33} \frac{\partial u_{z i}}{\partial z}+e_{33} \frac{\partial \phi_{i}}{\partial z}-\lambda_{33} T_{i} \\
\sigma_{z x i} & =c_{44}\left(\frac{\partial u_{x i}}{\partial z}+\frac{\partial u_{z i}}{\partial x}\right)+e_{15} \frac{\partial \phi_{i}}{\partial x}
\end{array}\right\}
$$

ここに，下添字 $i=0,1,2$ はそれぞれ $-h_{0} \leq z \leq h_{0}$, $h_{0} \leq z \leq h_{1}, \quad-h_{2} \leq z \leq-h_{0}$ の領域の場を表寸.

熱伝導方程式惊次式となる。

$$
\kappa^{2} \frac{\partial^{2} T_{i}}{\partial x^{2}}+\frac{\partial^{2} T_{i}}{\partial z^{2}}=0 \quad(i=0,1,2)
$$

ここに, $\kappa^{2}=\kappa_{x} / \kappa_{z}$ である。また，文献 (17) を考虑 すると変位掞よび静電ポテンシャルに関する場の支配 方程式は

$$
\left.\begin{array}{l}
c_{11} \frac{\partial^{2} u_{x i}}{\partial x^{2}}+c_{44} \frac{\partial^{2} u_{x i}}{\partial z^{2}}+\left(c_{13}+c_{44}\right) \frac{\partial^{2} u_{z i}}{\partial x \partial z} \\
+\left(e_{31}+e_{15}\right) \frac{\partial^{2} \phi_{i}}{\partial x \partial z}=\lambda_{11} \frac{\partial T_{i}}{\partial x} \\
c_{44} \frac{\partial^{2} u_{z i}}{\partial x^{2}}+c_{33} \frac{\partial^{2} u_{z i}}{\partial z^{2}}+\left(c_{13}+c_{44}\right) \frac{\partial^{2} u_{x i}}{\partial x \partial z} \\
+e_{15} \frac{\partial^{2} \phi_{i}}{\partial x^{2}}+e_{33} \frac{\partial^{2} \phi_{i}}{\partial z^{2}}=\lambda_{33} \frac{\partial T_{i}}{\partial z} \\
e_{15} \frac{\partial^{2} u_{z i}}{\partial x^{2}}+e_{33} \frac{\partial^{2} u_{z i}}{\partial z^{2}}+\left(e_{15}+e_{31}\right) \frac{\partial^{2} u_{x i}}{\partial x \partial z} \\
-\varepsilon_{11} \frac{\partial^{2} \phi_{i}}{\partial x^{2}}-\varepsilon_{33} \frac{\partial^{2} \phi_{i}}{\partial z^{2}}=-p_{z} \frac{\partial T_{i}}{\partial z}
\end{array}\right\}
$$

本問題の温度場に関寸る境界条件式は

$$
\begin{aligned}
& \left.\begin{array}{ll}
\partial T_{0}\left(x, \theta_{0 i}\right) / \partial z=0 & \left(|x|<c_{i}\right) \\
T_{0}\left(x \cdot \theta_{0 i}\right)=T_{i}\left(x, \theta_{0 i}\right) & \left(|x| \geq c_{i}\right)
\end{array}\right\}(i=1.2) \\
& \left.\partial T_{0}\left(x, \theta_{0 i}\right) / \partial z=\partial T_{i}\left(x, \theta_{0 i}\right) / \partial z \quad(|x| \geq 0)\right\} \\
& T_{i}\left(x, \theta_{1 i}\right)=T_{i 0} \\
& (|x| \geq 0)\} \\
& (i=1,2)
\end{aligned}
$$

また, 電気弾性場に関寸万境界条件式は (15)

$$
\left.\begin{array}{ll}
\sigma_{z z 0}\left(x, \theta_{0 i}\right)=0 & \left(|x|<c_{i}\right) \\
u_{z 0}\left(x, \theta_{0 i}\right)=u_{z i}\left(x, \theta_{0 i}\right) & \left(|x| \geq c_{i}\right)
\end{array}\right\}(i=1,2)
$$

\section{3. 温度場解析}

温度場 $T_{i}(x, z)(i=0,1,2)$ を次のように置く.

$$
T_{i}(x, z)=T^{(1)}(z)+T_{i}^{(2)}(x, z) \quad(i=0,1,2)
$$

ここに, 非擾乱温度場 $T^{(1)}(z)$ は前報 (11) と同様で, 以下のようになる。

$$
T^{(1)}(z)=\frac{1}{h}\left[T_{10} h_{2}+T_{20} h_{1}+\left(T_{10}-T_{20}\right) z\right]
$$

また, 擾乱温度場 $T_{i}^{(2)}(x, z)(i=0,1,2)$ に関する支 配方程式および境界条件式は

$$
\kappa^{2} \frac{\partial^{2} T_{i}^{(2)}}{\partial x^{2}}+\frac{\partial^{2} T_{i}^{(2)}}{\partial z^{2}}=0 \quad(i=0,1,2)
$$

$$
\left.\begin{array}{ll}
\partial T_{0}^{(2)}\left(x, \theta_{0 i}\right) / \partial z=-d T^{(1)}\left(\theta_{0 i}\right) / d z & \left(|x|<c_{i}\right) \\
T_{0}^{(2)}\left(x, \theta_{0 i}\right)=T_{i}^{(2)}\left(x, \theta_{0 i}\right) & \left(|x| \geq c_{i}\right)
\end{array}\right\}
$$


式 (15) に Fourier 変換法を適用して擾乱温度場の一 般解を求め万と(12)

$$
\begin{array}{r}
T_{i}^{(2)}(x . z)=\frac{1}{2 \pi} \sum_{j=1}^{2} \int_{-\infty}^{\infty} D_{i, j}(s) \exp \left(|s| \kappa_{i, j} z\right) \\
\times \exp (-\mathrm{i} s . x) d s(i=0,1,2)
\end{array}
$$

ここに, $D_{i j}(s)(i=0,1,2, j=1.2)$ は境界条件式より 決定される未知関数であり, $\kappa_{i j}(i=0,1,2, j=1,2)$ は

$$
\left.\begin{array}{l}
\kappa_{i 1}=-k \\
\kappa_{i 2}=\kappa
\end{array}\right\}(i=0,1,2)
$$

新しい未知関数 $G_{k 00}(x)(k=1.2)$ を次のように導 入す万(13).

$G_{k 0}(x)=\left\{\begin{array}{ll}\frac{\partial}{\partial x}\left[T_{0}^{(2)}\left(x, \theta_{0 k}\right)-T_{k}^{(2)}\left(x, \theta_{0 k}\right)\right] & \left(|x|<c_{k}\right) \\ 0 & \left(|x| \geq c_{k}\right)\end{array}\right\}$ $(k=1,2) \quad(20)$

従って，境界条件式 (17) を考慮寸ると，混合境界条 件式 (16) の第 1 式より，未知関数 $G_{10}(t), G_{20}(\mu t)$ に 関寸る次の連立特異積分方程式が得られる。

$$
\begin{aligned}
& \frac{\kappa}{2 \pi} \int_{-c}^{c}\left\{\left[\frac{1}{t-x}+M_{011}\right] G_{10}(t)\right. \\
& \left.-\mu M_{012} G_{20}(\mu t)\right\} d t=\frac{T_{10}-T_{20}}{h} \\
& \frac{\kappa}{2 \pi} \int_{-c}^{c}\left\{M_{021} G_{10}(t)\right. \\
& \left.\left.-\left[\frac{1}{t-x}+\mu M_{022}\right] G_{20}(\mu t)\right\} d t=\frac{T_{10}-T_{20}}{h}\right\} \\
& \quad[|x|<c) \quad(21)
\end{aligned}
$$

式中の積分核 $M_{0 j k}=M_{0 j k}(t, x)(j, k=1,2)$ 仗次式 で与えられ\%。

$$
\begin{aligned}
& M_{011}=\int_{0}^{\infty}\left\{\rho_{1}(s)\left\{1+\exp \left[-2 s \kappa\left(h_{2}+h_{0}\right)\right]\right\}-1\right\} \\
& \times \sin [s(t-x)] d s \\
& M_{012}=\int_{0}^{\infty} \rho_{2}(s)\left[\exp \left(-2 s \kappa h_{0}\right)+\exp \left(-2 s \kappa h_{1}\right)\right] \\
& \times \sin [s(\mu t-x)] d s \\
& M_{021}=\int_{0}^{\infty} \rho_{1}(s)\left[\exp \left(-2 s \kappa h_{0}\right)+\exp \left(-2 s \kappa h_{2}\right)\right] \\
& \times \sin [s(t-\mu x)] d s \\
& \left.\begin{array}{rl}
M_{022}=\int_{0}^{\infty} & \left\{\rho_{2}(s)\left\{1+\exp \left[-2 s \kappa\left(h_{1}+h_{0}\right)\right]\right\}-1\right\} \\
& \times \sin [s \mu(t-x)] d s
\end{array}\right\}
\end{aligned}
$$

$\Xi こ に$

$$
\rho_{j}(s)=\frac{1+\exp \left[-2 s \kappa\left(h_{j}-h_{0}\right)\right]}{1-\exp (-2 s k h)} \quad(j=1.2)
$$

また，混合境界条件式 (16) の第 2 式より，次の補足 の条件式が得られる。

$$
\int_{-c}^{c} G_{k 0}\left(t^{\prime}\right) d t^{\prime}=0 \quad(k=1,2)
$$

$こ こ に$

$$
t^{\prime}=\left\{\begin{array}{ll}
t & (k=1) \\
\mu t & (k=2)
\end{array}\right\}
$$

特異積分方程式 (21) および補足の条件式 (24) を Gauss-Jocobi の数值積分公式 (14) を用いて数值解析 することにより， $G_{k 0}\left(t^{\prime}\right)(k=1,2)$ が求まる.さらに, 式 (18) の未知関数 $D_{i j}(s)(i=0.1,2, j=1,2)$ を得 られた $G_{k 0}\left(t^{\prime}\right)(k=1.2)$ を用いて表すと, 擾乱温度 場 $T_{i}^{(2)}(x, z)(i=0,1,2)$ が次のように決定される.

$$
\begin{aligned}
& T_{i}^{(2)}(x, z)=-\frac{1}{\pi} \sum_{j=1}^{2} \sum_{k=1}^{2} \int_{0}^{\infty} \frac{1}{s} R_{i, j k}(s) \\
& \times \exp \left[s\left(\kappa_{i j} z+\kappa h_{i j}\right)\right] d s \\
& \times \int_{-c}^{c}\left(G_{k 00}\left(t^{\prime}\right) \sin \left[s\left(t^{\prime}-x\right)\right] d t^{\prime} \quad(i=0,1,2)(20)\right.
\end{aligned}
$$

式中の関数 $R_{i j k}(s)$ および定数 $h_{i j}(i=0,1,2, j, k=$ 1,2) はそれぞれ付録 1 に示寸。

\section{4. 電気熱弾性場解析}

非擾乱温度場 $T^{(1)}(z)$ は, 特異電気弾性場を誘起し ないため), 携乱温度場 $T_{i}^{(2)}(x, z)(i=0,1,2)$ に起因寸 万電気熱弾性場に注目する。変位ベクトルの成分 $u_{z i}$ $=u_{z i}(x, z), u_{x i}=u_{x i}(x, z)$ および静電ポテンシャル $\phi_{i}=\phi_{i}(x, z)(i=0,1,2)$ を次式で表示.

$$
\left.\begin{array}{l}
u_{z i}=u_{z i}^{(1)}+u_{z i}^{(2)} \\
u_{x i}=u_{x i}^{(1)}+u_{x i}^{(2)} \\
\phi_{i}=\phi_{i}^{(1)}+\phi_{i}^{(2)}
\end{array}\right\}(i=0,1,2)
$$

ここに, $u_{z i}^{(1)}=u_{z i}^{(1)}(x, z), \quad u_{x i}^{(1)}=u_{x i}^{(1)}(x, z), \phi_{i}^{(1)}=$ $\phi_{i}^{(1)}(x, z)$ は式 (4) における温度場 $T_{i}(x, z)$ を擾乱温 度場 $T_{i}^{(2)}(x, z)$ とした非同次方程式の特解, $u_{z i}^{(2)}=$ $u_{z i}^{(2)}(x, z), u_{x i}^{(2)}=u_{x i}^{(2)}(x, z), \phi_{i}^{(2)}=\phi_{i}^{(2)}(x, z)(i=$ $0,1,2)$ 付式 (4) に拈いて温度場を零とした同次方程式 の一般解である.

特解は熱弾性変位ポテンシャル法により, 次のよう に求まる。 
$u_{z i}^{(1)}=\frac{1}{2 \pi} \sum_{j=1}^{2} \int_{-\infty}^{\infty} \frac{p_{4 i j}^{(1)} F_{i j}(s)}{s|s|} \exp \left[|s|\left(\kappa_{i j} z+\kappa h_{i j}\right)\right]$

$$
\times \exp (-\mathrm{i} s x) d s
$$

$u_{x i}^{(1)}=\frac{1}{2 \pi} \sum_{j=1}^{2} \int_{-\infty}^{\infty} \frac{p_{5 i j}^{(1)} F_{i j}(s)}{s^{2}} \exp \left[|s|\left(\kappa_{i j} z+\kappa h_{i j}\right)\right]$ $\times \exp (-\mathrm{i} s x) d s$

$(i=0,1,2)$

$\phi_{i}^{(1)}=-\frac{1}{2 \pi} \sum_{j=1}^{2} \int_{-\infty}^{\infty} \frac{p_{6 i j}^{(1)} F_{i j}(s)}{s|s|} \exp \left[|s|\left(\kappa_{i j} z+\kappa h_{i, j}\right)\right]$ $\times \exp (-\mathrm{i} s x) d s$

ここに, 定数 $p_{m i j}^{(1)}(m=4,5,6, i=0,1.2, j=1,2)$ は前報 $(11)$ の付録 2 と同様であり, 関数 $F_{i j}(s)(i=$ $0,1,2, j=1,2)$ は

$$
\begin{array}{r}
F_{i j}(s)=\sum_{k=1}^{2} R_{i j k}(s) \int_{-c}^{c} G_{k 0}\left(t^{\prime}\right) \exp \left(\mathrm{ist} t^{\prime}\right) d t^{\prime} \\
(i=0,1,2, j=1,2)
\end{array}
$$

また,一般解は Fourier 変換法により, 次のように 求まる(12).

$$
\begin{aligned}
u_{z i}^{(2)}= & \frac{\mathrm{i}}{2 \pi} \sum_{j=1}^{6} \int_{-\infty}^{\infty} p_{4 i j}^{(2)} A_{i j}(s) \exp \left(|s| \gamma_{i j} z\right) \\
& \times \exp (-\mathrm{i} s x) d s \quad(i=0,1,2) \quad(32) \\
u_{x i}^{(2)}= & \frac{\mathrm{i}}{2 \pi} \sum_{j=1}^{6} \int_{-\infty}^{\infty} \frac{|s|}{s} p_{5 i j}^{(2)} A_{i j}(s) \exp \left(|s| \gamma_{i j} z\right) \\
& \times \exp (-\mathrm{i} s x) d s \quad(i=0,1,2) \quad(33) \\
\phi_{i}^{(2)}= & -\frac{\mathrm{i}}{2 \pi} \sum_{j=1}^{6} \int_{-\infty}^{\infty} p_{6 i j}^{(2)} A_{i j}(s) \exp \left(|s| \gamma_{i j} z\right) \\
& \times \exp (-\mathrm{i} s x) d s \quad(i=0,1,2) \quad(34)
\end{aligned}
$$

ここに, $A_{i j}(s)(i=0,1,2, j=1,2, \ldots, 6)$ 以境界条 件式より決定される未知関数であり, 定数 $p_{m i j}^{(2)}(m=$ $4,5,6, i=0,1,2, j=1,2, \ldots, 6)$ は什録 2 に示寸.

温度場解析之同㥞に, 問題の解を特異積分方程式 の解に導くため, 次の新しい未知関数 $G_{k m}(x)(k=$ $1,2, m=1,2,3)$ を導入する ${ }^{(13)}$.

$G_{k 1}(x)=\left\{\begin{array}{ll}\frac{\partial}{\partial x}\left[u_{z 0}\left(x, \theta_{0 k}\right)-u_{z k}\left(x, \theta_{0 k}\right)\right] & \left(|x|<c_{k}\right) \\ 0 & \left(|x| \geq c_{k}\right)\end{array}\right\}$

$$
(k=1,2) \quad(35)
$$

$G_{k 2}(x)=\left\{\begin{array}{ll}\frac{\partial}{\partial x}\left[u_{x 0}\left(x, \theta_{0 k}\right)-u_{x k}\left(x, \theta_{0 k}\right)\right] & \left(|x|<c_{k}\right) \\ 0 & \left(|x| \geq c_{k}\right)\end{array}\right\}$

$(k=1,2) \quad(36)$

$G_{k 3}(x)=\left\{\begin{array}{ll}-\frac{\partial}{\partial x}\left[\phi_{0}\left(x, \theta_{0 k}\right)-\phi_{k}\left(x, \theta_{0 k}\right)\right] & \left(|x|<c_{k}\right) \\ 0 & \left(|x| \geq c_{k}\right)\end{array}\right\}$

$(k=1,2) \quad(37)$
境界条件式 (11)，(12) を考虑寸当と，混合境界条件式 $(8) \sim(10)$ の第 1 式より, 未知関数 $G_{k m}\left(t^{\prime}\right)(k=1.2$, $m=1,2.3)$ に関寸る 6 本の連立特異積分方程式が得 られる。

$$
\begin{aligned}
& \frac{1}{\pi} \int_{-c}^{c}\left\{\left[\frac{Z_{1111}^{(1) \infty}}{t-x}+M_{111}^{(1)}\right] G_{11}\left(t^{\prime}\right)+M_{112}^{(1)} G_{12}\left(t^{\prime}\right)\right. \\
& \left.+\left[\frac{Z_{1131}^{(1) \infty}}{t-x}+M_{113}^{(1)}\right] G_{13}\left(t^{\prime}\right)+\mu \sum_{m=1}^{3} M_{12 m}^{(1)} G_{2 m}\left(t^{\prime}\right)\right\} d t \\
& =\sigma_{z z 0}^{T}\left(x^{\prime}, h_{0}\right) \quad(|x|<c) \quad(38)
\end{aligned}
$$

$\frac{1}{\pi} \int_{-c}^{c}\left\{M_{211}^{(1)} G_{11}\left(t^{\prime}\right)+\left[\frac{Z_{2121}^{(1) \infty}}{t-x}+M_{212}^{(1)}\right] G_{12}\left(t^{\prime}\right)\right.$

$\left.+M_{213}^{(1)} G_{13}\left(t^{\prime}\right)+\mu \sum_{m=1}^{3} M_{22 m}^{(1)} G_{2 m}\left(t^{\prime}\right)\right\} d t$

$=\sigma_{z x 0}^{T}\left(x^{\prime}, h_{0}\right)$

$(|x|<c) \quad(39)$

$\frac{1}{\pi} \int_{-c}^{c}\left\{\left[\frac{Z_{311}^{(1) \infty}}{t-x}+M_{311}^{(1)}\right] G_{11}\left(t^{\prime}\right)+M_{312}^{(1)} G_{12}\left(t^{\prime}\right)\right.$

$\left.+\left[\frac{Z_{3131}^{(1) \infty}}{t-x}+M_{313}^{(1)}\right] G_{13}\left(t^{\prime}\right)+\mu \sum_{m=1}^{3} M_{32 m}^{(1)} G_{2 m}\left(t^{\prime}\right)\right\} d t$

$=D_{z 0}^{T}\left(x^{\prime}, h_{0}\right)+D_{0}$

$(|x|<c) \quad(40)$

$\frac{1}{\pi} \int_{-c}^{c}\left\{\sum_{m=1}^{3} M_{11 m}^{(2)} G_{1 m}\left(t^{\prime}\right)+\left[\frac{Z_{1211}^{(2) \infty}}{t-x}+\mu M_{121}^{(2)}\right] G_{21}\left(t^{\prime}\right)\right.$

$\left.+\mu M_{122}^{(2)} G_{22}\left(t^{\prime}\right)+\left[\frac{Z_{1231}^{(2) \infty}}{t-x}+\mu M_{123}^{(2)}\right] G_{23}\left(t^{\prime}\right)\right\} d t$

$=\sigma_{z z 0}^{T}\left(x^{\prime},-h_{0}\right)$

$(|x|<c) \quad(41)$

$\frac{1}{\pi} \int_{-c}^{c}\left\{\sum_{m=1}^{3} M_{21 m}^{(2)} G_{1 m}\left(t^{\prime}\right)+\mu M_{221}^{(2)} G_{21}\left(t^{\prime}\right)\right.$

$\left.+\left[\frac{Z_{2221}^{(2) \infty}}{t-x}+\mu M_{222}^{(2)}\right] G_{22}\left(t^{\prime}\right)+\mu M_{223}^{(2)} G_{23}\left(t^{\prime}\right)\right\} d t$

$=\sigma_{z x 0}^{T}\left(x^{\prime},-h_{0}\right)$

$\frac{1}{\pi} \int_{-c}^{c}\left\{\sum_{m=1}^{3} M_{31 m}^{(2)} G_{1 m}\left(t^{\prime}\right)+\left[\frac{Z_{3211}^{(2) \infty}}{t-x}+\mu M_{321}^{(2)}\right] G_{2 \ell}\left(t^{\prime}\right)\right.$ $\left.+\mu M_{322}^{(2)} G_{22}\left(t^{\prime}\right)+\left[\frac{Z_{3231}^{(2) \infty}}{t-x}+\mu M_{323}^{(2)}\right] G_{23}\left(t^{\prime}\right)\right\} d t$

$$
=D_{z 0}^{T}\left(x^{\prime},-h_{0}\right)+D_{0}
$$

$(|x|<c)$

式中の定数 $Z_{j k m l}^{(n) \infty}(j, m=1,2,3, n, k, l=1,2)$ 住 前報 (11) の付録 3 と同様であり, 積分核 $M_{j k m}^{(n)}=$ $M_{j k m}^{(n)}\left(t^{\prime}, x^{\prime}\right)(j, m=1,2,3, k=1,2)$ は次式で与 えられる。 
$M_{. j k m}^{(n)}=$

$\left.\left\{\begin{array}{rr}\int_{0}^{\infty} \sum_{l=1}^{2}\left[Z_{j k m l}^{(n)}(s)-Z_{j k m l}^{(n) \infty}\right] & \sin \left[s\left(t^{\prime}-x^{\prime}\right)\right] d s \\ (m=1,3) \\ -\int_{0}^{\infty} \sum_{l=1}^{2}\left[Z_{j k m l}^{(n)}(s)-Z_{j k m l}^{(n) \infty}\right] & \cos \left[s\left(t^{\prime}-x^{\prime}\right)\right] d s \\ (m=2)\end{array}\right\}\right)$

$(n, k=1,2)$

ここに,

$$
x^{\prime}=\left\{\begin{array}{ll}
x & (n=1) \\
\mu x & (n=2)
\end{array}\right\}
$$

また， $\sigma_{z z 0}^{T}\left(x^{\prime}, \pm h_{0}\right), \sigma_{z x 0}^{T}\left(x^{\prime}, \pm h_{0}\right)$ および $D_{z 0}^{T}\left(x^{\prime}\right.$, $\left.\pm h_{0}\right)$ は, 擾乱温度場 $T^{(2)}(x, z)$ に起因し, き裂が存 在しない厚板の $z= \pm h_{0}$ 面上での熱応力・電束密度 成分に相当寸るもので，次のように得られる。

$$
\begin{array}{r}
\sigma_{z z 0}^{T}\left(x^{\prime}, \pm h_{0}\right)=\lim _{z \rightarrow \pm h_{0}} \frac{p_{10 j}^{(2)}}{2 \pi} \sum_{j=1}^{6} \int_{-\infty}^{\infty}|s| \Gamma_{1 j} \exp (-\mathrm{i} s x) d s \\
-\lim _{z \rightarrow \pm h_{0}} \frac{\mathrm{i} p_{10 j}^{(1)}}{2 \pi} \sum_{j=1}^{2} \int_{-\infty}^{\infty} \frac{1}{s} \Gamma_{2 j} \exp (-\mathrm{i} s x) d s \quad(46) \\
\sigma_{z x 0}^{T}\left(x^{\prime}, \pm h_{0}\right)=\lim _{z \rightarrow \pm h_{0}} \frac{\mathrm{i} p_{20 j}^{(2)}}{2 \pi} \sum_{j=1}^{6} \int_{-\infty}^{\infty} s \Gamma_{1 j} \exp (-\mathrm{i} s x) d s \\
+\lim _{z \rightarrow \pm h_{0}} \frac{p_{20 j}^{(1)}}{2 \pi} \sum_{j=1}^{2} \int_{-\infty}^{\infty} \frac{1}{|s|} \Gamma_{2 j} \exp (-\mathrm{i} s x) d s \quad(47) \\
D_{z 0}^{T}\left(x^{\prime}, \pm h_{0}\right)=\lim _{z \rightarrow \pm h_{0}} \frac{p_{30 j}^{(2)}}{2 \pi} \sum_{j=1}^{6} \int_{-\infty}^{\infty}|s| \Gamma_{1 j} \exp (-\mathrm{i} s x) d s \\
-\lim _{z \rightarrow \pm h_{0}} \frac{\mathrm{i} p_{30 j}^{(1)}}{2 \pi} \sum_{j=1}^{2} \int_{-\infty}^{\infty} \frac{1}{s} \Gamma_{2 j} \exp (-\mathrm{i} s x) d s \quad(48)
\end{array}
$$

式中の $\Gamma_{k j}=\Gamma_{k j}(s)(k=1,2, j=1,2, \ldots, 6)$ は前 報 (11) の式 (42) 中の $F_{i j}(s)(i=0,1,2, j=1,2)$ を 式 (31) に置き換えたものと同様である。また，混合 境界条件式 $(8) \sim(10)$ の第 2 式より, 次の補足の条件 式が得られる。

$$
\int_{-c}^{c} G_{k m}\left(t^{\prime}\right) d t^{\prime}=0 \quad(k=1,2, m=1,2,3)
$$

特異積分方程式 (21), (38) (43) および補足の条件 式 (24),(49) をGauss-Jacobi O数值積分公式 (14) を用 いて数值解析寸るために，解の特暴性を考慮し， $G_{k m}$ $\left(t^{\prime}\right)$ を滑らかな関数 $\Phi_{k m}\left(t^{\prime}\right)(k=1,2, m=0.1,2.3)$ を用いて次のように置く.

$$
\begin{array}{r}
G_{k m}\left(t^{\prime}\right)=\frac{c_{k}}{\left[\left(c_{k}+t^{\prime}\right)\left(c_{k}-t^{\prime}\right)\right]^{1 / 2}} \Phi_{k m}\left(t^{\prime}\right) \\
(k=1,2, m=0,1,2,3)
\end{array}
$$

従って, き裂先端の特異挙動を評価寸万と ${ }^{(18)}$, 応力 拡大係数 $K_{I}^{ \pm}, K_{I I}^{ \pm}$および電束密度拡大係数 $K_{D}^{ \pm}$が次 のように得られる。

$\left.\begin{array}{l}K_{I}^{+}=\left(\pi c_{k}\right)^{1 / 2}\left[Z_{1111}^{\infty} \Phi_{11}\left(c_{k}\right)+Z_{1131}^{\infty} \Phi_{13}\left(c_{k}\right)\right] \\ K_{I I}^{+}=\left(\pi c_{k}\right)^{1 / 2} Z_{2121}^{\infty} \Phi_{12}\left(c_{k}\right) \\ K_{D}^{+}=\left(\pi c_{k}\right)^{1 / 2}\left[Z_{3111}^{\infty} \Phi_{11}\left(c_{k}\right)+Z_{3131}^{\infty} \Phi_{13}\left(c_{k}\right)\right]\end{array}\right\}$

$K_{I}^{-}=\left(\pi c_{k}\right)^{1 / 2}\left[Z_{1211}^{\infty} \Phi_{21}\left(c_{k}\right)+Z_{1231}^{\infty} \Phi_{23}\left(c_{k}\right)\right]$

$K_{I I}^{-}=\left(\pi c_{k}\right)^{1 / 2} Z_{2221}^{\infty} \Phi_{22}\left(c_{k}\right)$

$\left.K_{D}^{-}=\left(\pi c_{k}\right)^{1 / 2}\left[Z_{3211}^{\infty} \Phi_{21}\left(c_{k}\right)+Z_{3231}^{\infty} \Phi_{23}\left(c_{k}\right)\right]\right\}$

ここに，上添字,+ 一 はそれぞれ $z=h_{0},-h_{0}$ 面上の き裂に関する諸量を示す。

\section{5. 数値結果および考察}

数值計算を行い, 特異電気熱弾性場に及ぼすき裂長 さ比 $c / h$, き裂長さの相異を表すパラメータ $\mu$, き裂 位置を表すパラメータ $h_{2} / h$, き裂間隔比 $h_{0} / h$ の影響 を解明する. 数值計算例には, 次に示寸セレン化カド ミウムの電気熱弾性特性を用いた ${ }^{(3)}$.

$$
\begin{aligned}
& c_{11}=7.41 \times 10^{10}[\mathrm{~Pa}], c_{33}=8.36 \times 10^{10}[\mathrm{~Pa}] \\
& c_{44}=1.32 \times 10^{10}[\mathrm{~Pa}], c_{13}=3.93 \times 10^{10}[\mathrm{~Pa}] \\
& e_{31}=-0.160\left[\mathrm{C} / \mathrm{m}^{2}\right], e_{33}=0.347\left[\mathrm{C} / \mathrm{m}^{2}\right] \\
& e_{15}=-0.138\left[\mathrm{C} / \mathrm{m}^{2}\right] \\
& \varepsilon_{11}=0.825 \times 10^{-10}[\mathrm{C} / \mathrm{Vm}] \\
& \varepsilon_{33}=0.903 \times 10^{-10}[\mathrm{C} / \mathrm{Vm}] \\
& \lambda_{11}=0.621 \times 10^{6}[\mathrm{~Pa} / \mathrm{K}] \\
& \lambda_{33}=0.551 \times 10^{6}[\mathrm{~Pa} / \mathrm{K}] \\
& p_{z}=-2.94 \times 10^{-6}\left[\mathrm{C} / \mathrm{Km}{ }^{2}\right]
\end{aligned}
$$

熱伝導率に関しては, $\kappa^{2}=\kappa_{x} / \kappa_{z}=1 / 1.5$ と仮定した.

まず, 温度場 $T_{i}(x, z)(i=0,1,2)$ について検討す る. 図 2 は, $h_{0} / h=0.2, h_{2} / h=0.5, c / h=0.5$ と した場合の $z= \pm h_{0}$ 面上における標準化された温度 分布 $\left(T_{i}-T_{20}\right) / T_{0}(i=0,1,2)$ を $\mu=0.5,1.0$ として 示したグラフであり, $T_{0}=T_{10}-T_{20}$ である. $\mu=1.0$ の場合, 対称性により $T_{1}^{(2)}\left(x, h_{0}\right)=-T_{2}^{(2)}\left(x,-h_{0}\right)$, $T_{0}^{(2)}\left(x, h_{0}\right)=-T_{0}^{(2)}\left(x,-h_{0}\right)$ となる. $\mu=0.5$ の場合, き裂上下面の温度差は上側のき裂では大となり下側の き裂では小となる。 


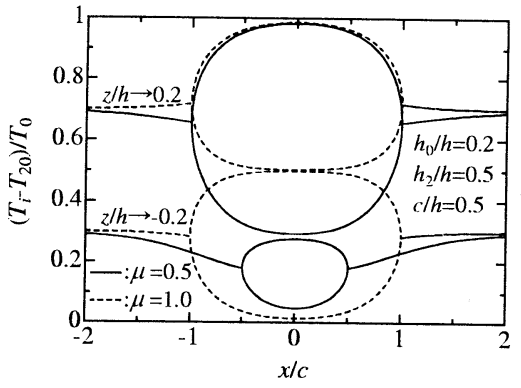

Fig.2 The temperatures on the crack faces and the crack extended lines.

次に，電気熱弾性場について検討寸る。図 $3,4,5$ は図 2 の温度場によって発生する $z= \pm h_{0}$ 面上に おける標準化された応力成分 $\left(\sigma_{z z 0}^{T} ， \sigma_{z, x 0}^{T}\right) / \lambda_{33} T_{0}$ お よび電束密度成分 $D_{z 0}^{T} / p_{z} T_{0}$ を示したグラフであ る. $\mu=1.0$ の場合, 対称性により, $\sigma_{z z 0}^{T}\left(x, h_{0}\right)=$ $-\sigma_{z z 0}^{T}\left(x,-h_{0}\right), \sigma_{z x 0}^{T}\left(x, h_{0}\right)=\sigma_{z x 0}^{T}\left(x,-h_{0}\right), D_{z 0}^{T}$ $\left(x, h_{0}\right)=-D_{z 0}^{T}\left(x,-h_{0}\right)$ となる. $z / h=-0.2$ の場 合, $\left[\left|\sigma_{z x 0}^{T}\left(x,-h_{0}\right)\right|\right]_{\mu=0.5}<\left[\left|\sigma_{z x 0}^{T}\left(x,-h_{0}\right)\right|\right]_{\mu=1.0}$ となるが, $\left[\left|\sigma_{z z 0}^{T}\left(x,-h_{0}\right)\right|,\left|D_{z 0}^{T}\left(x,-h_{0}\right)\right|\right]_{\mu=0.5}>$ $\left[\left|\sigma_{z z 0}^{T}\left(x,-h_{0}\right)\right|,\left|D_{z 0}^{T}\left(x,-h_{0}\right)\right|\right]_{\mu=10}$ となる. $\mu=0.5$ の場合，上側のき裂上下面の温度差は大となり， $\sigma_{z z 0}^{T}\left(x, h_{0}\right), \sigma_{z x 0}^{T}\left(x, h_{0}\right)$ および $D_{z 0}^{T}\left(x, h_{0}\right)$ の絶対值は $\mu=1.0$ の場合と比べ比較的大となる.

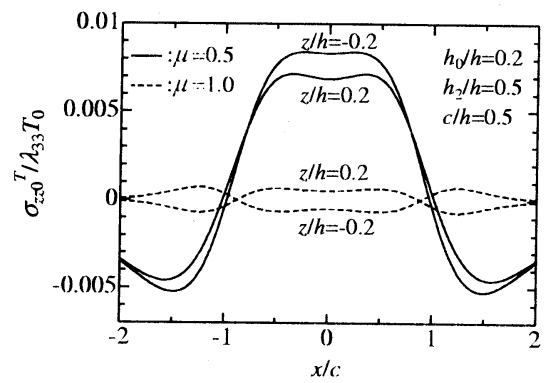

Fig.3 $\sigma_{z z 0}^{T}$ due to the temperatures shown in Fig. 2 .

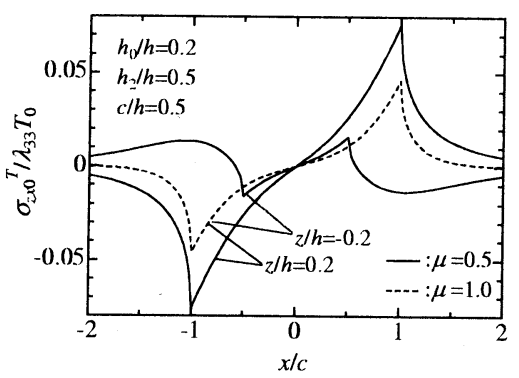

Fig.4 $\sigma_{z x 0}^{T}$ due to the temperatures shown in Fig. 2.

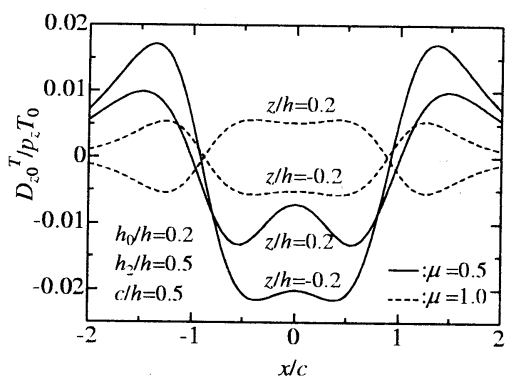

Fig.5 $D_{z 0}^{T}$ due to the temperatures shown in Fig.2.

最後に, 応力・電束密度桩大係数に及ぼ寸幾何学的形 状および電気的熱的負荷の影響を検討寸る. 図 6 は, 電 気的負荷のみが作用する場合の $\left(K_{I}^{ \pm}, K_{I}^{ \pm}\right) / \sigma_{0}(\pi c)^{1 / 2}$ および $K_{D}^{ \pm} / D_{0}(\pi c)^{1 / 2}$ に及ぼす $\mu$ の影響を示したグ ラフであり, $h_{0} / h=0.2, h_{2} / h=0.5, c / h=0.5$ と した.ここで, $\sigma_{0}=-D_{0} e_{33} / \varepsilon_{33}$ である. $\mu$ の増加 に伴い, $K_{D}^{-}$は単調に増大し, $K_{I}^{ \pm}$は $0.7<\mu<1.0$ で急激に変化する， $\mu=0.0$ の場合は，前報 ${ }^{(16)}$ の結 果と一致する. また, $\mu=1.0$ の場合, 対称性により $K_{I}^{+}=K_{I}^{-}, K_{I I}^{+}=-K_{I I}^{-}, K_{D}^{+}=K_{D}^{-}$となり, 二個 の等長き裂の結果 (11) と一致する.

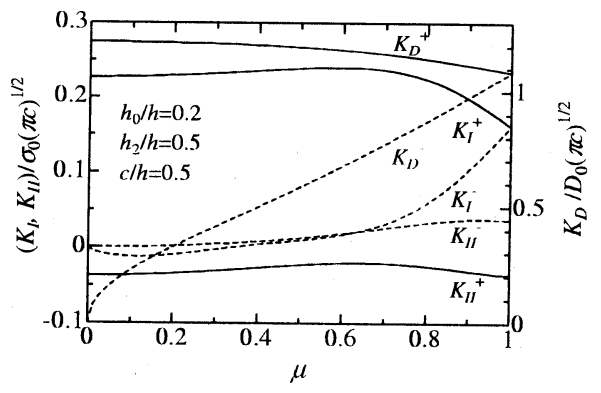

Fig.6 The effect of $\mu$ on the stress and electric displacement intensity factors under pure electrical load.

図 7〜10により, 熱的負㴕のみが作用する場命 $\left(D_{0} / p_{z} T_{0}=0.0\right)$ について検討寸る. 図 7 は $K_{I I}^{ \pm} / \lambda_{33}$ $T_{0}(\pi c)^{1 / 2}$ に及ぼす $c / h$ の影響を示したグラフであり， $h_{0} / h=0.2, h_{2} / h=0.5$ とした. $\mu=0.5$ の場含, $c / h$ の増加に伴い, $K_{I I}^{+} / \lambda_{33} T_{0}(\pi c)^{1 / 2}$ は単調に増大寸る が, $K_{I I}^{-} / \lambda_{33} T_{0}(\pi c)^{1 / 2}$ は増大し，最大值をとった後減 少する. $\mu=0.0,1.0$ の場合, 前報 (6),(11) の結果とそ れぞれ一致する。

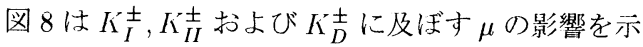
したもので, $h_{0} / h=0.2, h_{2} / h=0.5, c / h=0.5$ とした。 $K_{I}^{+}, K_{I I}^{+}$およ゙ $\left|K_{D}^{+}\right|$は $\mu$ の増加に伴い减 少する傾向を示寸. $K_{I I}^{-}$は $\mu$ の増加に伴って増大し, 
$K_{I}^{-} \cdot\left|K_{D}^{-}\right|$はそれぞれ $\mu \approx 0.75 .0 .54$ で最大值をとる. $\mu=1.00$ 場合, 対称性より $K_{I}^{+}=-K_{I}^{-}, K_{I I}^{+}=K_{I I}^{-}$, $K_{D}^{+}=-K_{D}^{-}$となる。

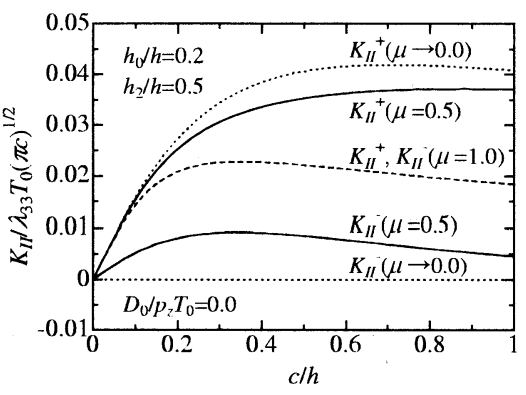

Fig.7 The effect of $c / h$ on the stress intensity factor $K_{I I}$.

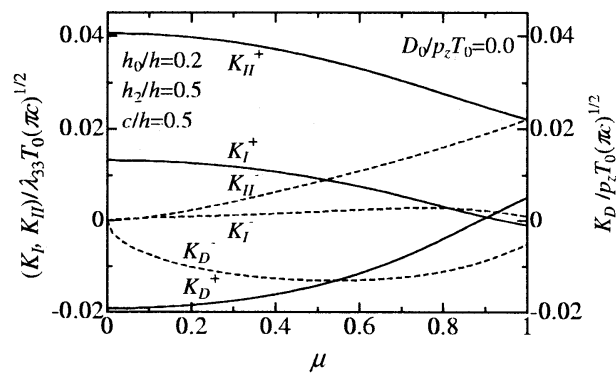

Fig.8 The effect of $\mu$ on the stress and electric displacement intensity factors.

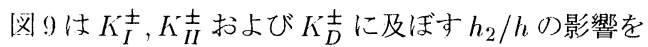
示したグラフであり, $h_{0} / h=0.2, c / h=0.5, \mu=0.5$ とした. $h_{2} / h$ の増加に伴い, $K_{I}^{ \pm}, K_{I I}^{-}$は単調に増大 寸るが, $K_{I I}^{+}, K_{D}^{ \pm}$は単調に減少寸る.

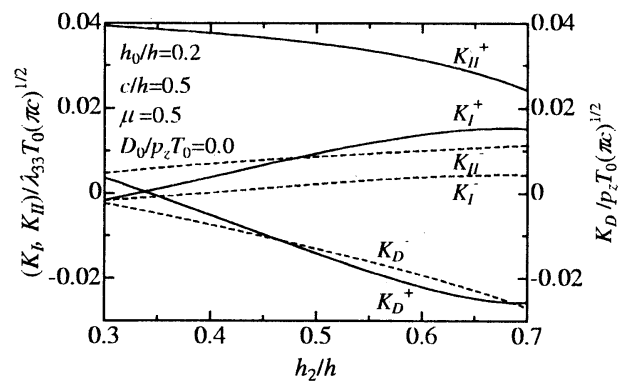

Fig.9 The effect of $h_{2} / h$ on the stress and electric displacement intensity factors.

図 10 は $K_{I}^{ \pm}, K_{I I}^{ \pm}$および $K_{D}^{ \pm}$に及ぼす $h_{0} / h$ の影響を 宗したグラフであり, $h_{2} / h=0.5, c / h=0.5, \mu=0.5$ とした.この場合の応力・電束密度拡大係数は $h_{0} / h$ に依存し複雑に変化する. $h_{0} / h$ の増加に伴い, $K_{I I}^{+}$, $\left|K_{D}^{-}\right|$は減少し, $K_{I}^{+},\left|K_{D}^{+}\right|$は増大寸る. しかし, き裂
が自由表面に接近寸るとそれぞれの絶対值は減少才る 傾问を示す。

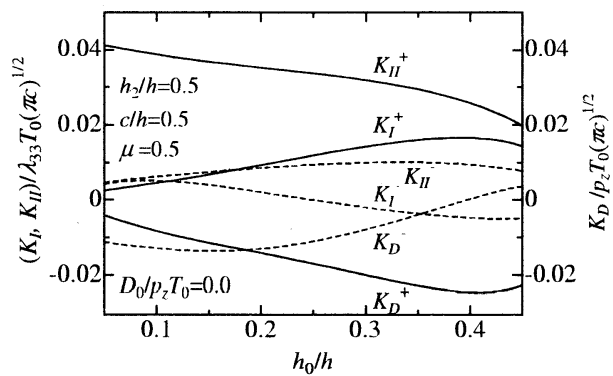

Fig.10 The effect of $h_{0} / h$ on the stress and electric displacement intensity factors.

\section{6. 結言}

本報は, 長さの異なる二個の平行き裂を有する圧電 厚板に電気的熱的負荷が作用寸る場合の応力・電束密 度拡大係数を理論解析したものである. また, 特別な 場合として単一き裂と二個の等長き裂の解も求め, 既 存解との比較を通して妥当性の検討も行った. 数值結 果より，(1) 標準化された応力・電束密度拡大係数は 幾何学的形状 $\left(c / h, \mu, h_{2} / h, h_{0} / h\right)$ により大きな影 響を受けること, (2) 二個の等長き裂 (11) と比較して 忍力・電束密度拡大係数に及ぼ寸き裂間相互干涉の影 響はより複雑になること，(3) き裂間隔が狭くなると $K_{I I}^{+}$が増大寸ることから，モードII型の破壊が予想さ れることなどが明らかになった。

\section{付録 1}

関数 $R_{i j k}(s)$ および定数 $h_{i j}(i=0,1,2, j, k=1,2)$ 仕

$$
\begin{aligned}
& R_{011}(s)=-\rho_{1}(s) \exp \left(-2 s \kappa h_{2}\right) / 2 \\
& R_{012}(s)=\rho_{2}(s) / 2, \quad R_{021}(s)=\rho_{1}(s) / 2 \\
& R_{022}(s)=-\rho_{2}(s) \exp \left(-2 s \kappa h_{1}\right) / 2 \\
& R_{11 k}(s)=-1 /\left\{1+\exp \left[-2 s \kappa\left(h_{1}-h_{0}\right)\right]\right\} \\
& \times\left[R_{02 k}(s)-R_{01 k}(s) \exp \left(-2 s \kappa h_{0}\right)\right] \\
& R_{22 k}(s)=-1 /\left\{1+\exp \left[-2 s \kappa\left(h_{2}-h_{0}\right)\right]\right\} \\
& \times\left[R_{01 k}(s)-R_{02 k}(s) \exp \left(-2 s \kappa h_{0}\right)\right] \\
& R_{12 k}(s)=-R_{11 k}(s), \quad R_{21 k}(s)=-R_{22 k}(s) \\
& (k=1,2) \\
& \left.\begin{array}{ll}
h_{01}=h_{02}=-h_{0}, & h_{11}=h_{22}=h_{0} \\
h_{12}=-2 h_{1}+h_{0}, & h_{21}=-2 h_{2}+h_{0}
\end{array}\right\}
\end{aligned}
$$

\section{付録 2}

定数 $p_{m i j}^{(2)}(m, j=1,2, \ldots, 6, i=0,1,2)$ は 


$$
\left.\begin{array}{r}
p_{1 i j}^{(2)}=c_{13} a_{i j}+\gamma_{i, j}\left(c_{33}-\epsilon_{33} b_{i j}\right) \\
p_{2 i j}^{(2)}=c_{44}\left(\gamma_{i j} a_{i j}-1\right)+\epsilon_{15} b_{i j} \\
p_{3 i j}^{(2)}=\epsilon_{31} a_{i, j}+\gamma_{i j}\left(e_{33}+\varepsilon_{33} b_{i j}\right) \\
p_{4 i, j}^{(2)}=-\mathrm{i}, \quad p_{5 i j}^{(2)}=a_{i j}, \quad p_{6 i j}^{(2)}=-\mathrm{i} b_{i, j}
\end{array}\right\}
$$

定数 $\gamma_{i j}(i=0,1,2, j=1,2, \ldots, 6)$ は, 次の特性方 程式の根である。

$$
\begin{aligned}
& \left(f_{4} g_{3}+g_{4} f_{3}\right) \gamma_{i, j}^{6}+\left(f_{4} g_{1}+f_{2} g_{3}+g_{4} f_{1}+g_{2} f_{3}\right) \gamma_{i, j}^{4} \\
& +\left(f_{2} g_{1}+f_{0} g_{3}+g_{2} f_{1}+g_{0} f_{3}\right) \gamma_{i j}^{2}+\left(f_{0} g_{1}+g_{0} f_{1}\right) \\
& =0 \quad(i=0,1,2, j=1,2, \ldots, 6) \\
& \text { ここに, } \operatorname{Re}\left[\gamma_{0 j}\right]<\operatorname{Re}\left[\gamma_{0 j+1}\right], \operatorname{Re}\left[\gamma_{1 j}\right]<\operatorname{Re}\left[\gamma_{1 j+1}\right] \text {, } \\
& \operatorname{Re}\left[\gamma_{2, j}\right]>\operatorname{Re}\left[\gamma_{2, j+1}\right](j=1,2, \ldots, 5) \text { であり } \\
& f_{4}=c_{44} e_{33}, \quad f_{0}=c_{11} \epsilon_{15} \\
& f_{3}=c_{33}\left(e_{15}+e_{31}\right)-e_{33}\left(c_{13}+c_{44}\right) \\
& f_{2}=\left(c_{13}+c_{44}\right)\left(e_{15}+e_{31}\right)-c_{11} e_{33}-c_{44} e_{15} \\
& f_{1}=-c_{44}\left(e_{15}+e_{31}\right)+e_{15}\left(c_{13}+c_{44}\right) \\
& g_{4}=c_{44} \varepsilon_{33}, \quad g_{0}=c_{11} \varepsilon_{11} \\
& g_{3}=e_{33}\left(e_{15}+e_{31}\right)+\varepsilon_{33}\left(c_{13}+c_{44}\right) \\
& g_{2}=-\left(e_{15}+e_{31}\right)^{2}-c_{11} \varepsilon_{33}-c_{44} \varepsilon_{11} \\
& g_{1}=-e_{15}\left(e_{15}+e_{31}\right)-\varepsilon_{11}\left(c_{13}+c_{44}\right)
\end{aligned}
$$

また, 定数 $a_{i j}, b_{i j}(i=0,1,2, j=1,2, \ldots, 6)$ は次式 で与えられる.

$$
\left.\begin{array}{c}
a_{i j}=\frac{g_{3} \gamma_{i j}^{3}+g_{1} \gamma_{i j}}{g_{4} \gamma_{i j}^{4}+g_{2} \gamma_{i j}^{2}+g_{0}} \\
b_{i j}=-\frac{\left(c_{44} \gamma_{i j}^{2}-c_{11}\right) a_{i j}-\left(c_{13}+c_{44}\right) \gamma_{i j}}{\gamma_{i j}\left(e_{15}+e_{31}\right)} \\
(i=0,1,2, j=1,2, \ldots, 6) \\
\text { 文 } \quad \text { 献 }
\end{array}\right\}
$$

\section{文}

(1) Rao, S. S. and Sunar, M., Piezoelectricity and Its Use in Disturbance Sensing and Control of Flexible Structures: A Survey, Applied Mechanics Review, Vol.47 (1994), pp.113-123.

(2) Noda, N. and Kimura, S., Deformation of a Piezothermoelectric Composite Plate, Journal of Thermal Stresses, Vol.21 (1998), pp.359-379.

(3) Ashida, F. and Tauchert, T. R., Transient Response of a Piezothermoelastic Circular Disk Under Axisymmetric Heating, Acta Mechanica., Vol.128 (1998), pp.1-14.

(4) Ootao, Y. and Tanigawa, Y., Three-Dimensional Transient Piezothermoelasticity in Functionally Graded Rectangular Plate Bonded to a Piezoelectric Plate, International Journal of Solids and Structures, Vol.37 (2000), pp.4377-4401.

(5) Wang, B. L. and Mai, Y. W., A Cracked Piezoelectric Material Strip under Transient Thermal Loading, Transactions of the ASME, Journal of Applied Mechanics, Vol.69 (2002), pp.539-546.
(6) Ueda. S. and Ikawa, K., The Crack Problem in Piezoelectric Strip under Thermoelectric Loading, Transactions of the Japan Society of Mechanical Engineers. Series A. Vol.72, No.715 (2006), pp.309-316.

(7) Ueda, S. et al., Thermal Stress Intensity Factors for a Normal Crack in a Semi-Infinite Piezoelectric Material, Transactions of the Japan Society of Mechanical Engineers, Series A, Vol.72, No.716 (2006), pp.513-519.

(8) Ueda, S. and Tani, Y., Thermal Stress Intensity Factors for a Normal Crack in a Piezoelectric Strip, Transactions of the Japan Society of Mechanical Engineers, Series A, Vol.72, No.722 (2006), pp.1487-1494.

(9) Chen, Z. T. and Worswick, M. J., Antiplane Mechanical and Inplane Electric Time-Dependent Load Applied to Two Coplanar Cracks in Piezoelectric Ceramic Material, Theoretical and Applied Fracture Mechanics, Vol.33, No.715 (2000), pp.173-184.

(10) Ueda, S. and Tani, Y., Thermal Stress Intensity Factors for Two Coplanar Cracks in a Piezoelectric Strip, Transactions of the Japan Society of Mechanical Engineers, Series A, Vol.73, No.736 (2007), pp.1410-1416.

(11) Ueda, S. and Ikawa, K., Thermoelectromechanical Interaction between Two Parallel Cracks in a Piezoelectric Strip, Transactions of the Japan Society of Mechanical Engineers, Series A, Vol.74, No.738 (2008), pp.42-49.

(12) Sneddon, I. N. and Lowengrub, M., Crack Problems in the Classical Theory of Elasticity, (1969), John Wiley \& Sons, Inc., New York.

(13) Erdogan, F. and Wu, B. H., Crack Problems in FGM Layers under Thermal Stresses, Journal of Thermal Stresses, Vol.19 (1996), pp.237-265.

(14) Erdogan, F. et al., Methods of Analysis and Solution of Crack Problems, (Edited by G.C.Sih), (1972), Noordhoff, Leyden.

(15) Wang, B. L. and Mai, Y. W., Impermeable Crack and Permeable Crack Assumptions, Which One Is More Realistic?, Transactions of the ASME, Journal of Applied Mechanics, Vol.71 (2004), pp.575-578.

(16) Ueda, S. et al., Electroelastic Response of a Parallel Crack in a Functionally Graded Piezoelectric Strip, Transactions of the Japan Society of Mechanical Engineers, Series A, Vol.72, No.716 (2006), pp.520-526.

(17) Hou, P. et al., A Point Heat Source on the Surface of a Semi-Infinite Transversely Isotropic Piezothermoelastic Material, Transactions of the ASME, Journal of Applied Mechanics, Vol.75 No.1 (2008), pp.011013.1-011013.8.

(18) Watanabe, K. et al., Mathematical Analyses in Elasticity for New Applications, (2007), pp.163-179, Yokendo. 